\title{
A SIMPLE TWISTED RELATIVE TRACE FORMULA
}

\author{
HEEKYOUNG HAHN
}

\begin{abstract}
In this article we derive a simple twisted relative trace formula.
\end{abstract}
\section{INTRODUCTION}

In this paper, we obtain a simple twisted relative trace formula analogous to the simple trace formula of Deligne and Kazhdan [BDKV] (see also [Ro]). We hope this simple twisted relative trace formula can be used to study distinguished admissible representations of a reductive group. A similar formula has already proved useful in the study of distinguished admissible representations of general linear groups [HM]. Our formula is also used crucially in [GW]. We will state the main result of [GW] after stating our main theorem.

Let $M / F$ be a quadratic or trivial extension of a number field $F$ and $\tau$ be the generator of $\operatorname{Gal}(M / F)$. Assume that $G_{0}$ is a connected reductive $F$-group with semisimple automorphism $\sigma$ of order 2 . We denote by $G_{0}^{\sigma}$ the group whose points in an $F$-algebra $R$ are given by

$$
G_{0}^{\sigma}(R)=\left\{g \in G_{0}(R): g^{\sigma}=g\right\} .
$$

Then $G_{0}^{\sigma}$ is reductive $[\mathrm{S} 2, \S 9]$. Write

$$
\begin{aligned}
G & :=\operatorname{Res}_{M / F} G_{0}, \\
G^{\sigma} & :=\operatorname{Res}_{M / F} G_{0}^{\sigma},
\end{aligned}
$$

set $\theta=\sigma \circ \tau$, and write $G^{\theta} \leq G$ for the subgroup whose points in an $F$-algebra $R$ are given by

$$
G^{\theta}(R)=\left\{g \in G(R): g^{\theta}=g\right\} .
$$

Note that $\sigma$ and $\tau$ commute.

In order to state our main result, we define the notion of an $F$-supercuspidal function.

Definition 1.1. Let $v$ be a finite place of $F$ and let $f_{v} \in C_{c}^{\infty}\left(G\left(F_{v}\right)\right)$. We say that $f_{v}$ is $F$-supercuspidal if any translate of $f_{v}$ (left or right) has zero integral along the unipotent radical of any proper parabolic of $G_{F_{v}}$ that is defined over $F$; i.e. is the base change of a parabolic subgroup of $G$.

Let

$$
\mu:=\mu_{1} \times \mu_{2}: G^{\sigma}\left(\mathbb{A}_{F}\right) \times G^{\theta}\left(\mathbb{A}_{F}\right) \longrightarrow \mathbb{C}^{\times}
$$

be a unitary character trivial on $G^{\sigma}(F) \times G^{\theta}(F)$. We now state our main result using notations that are defined precisely in $\S 2$ below.

Theorem 1.2. Suppose that $G^{\sigma}$ is connected. Let $f=\otimes_{v} f_{v} \in C_{c}^{\infty}\left(G\left(\mathbb{A}_{F}\right)\right)$ be such that there exist places $v_{1}, v_{2}, v_{3}$ of $F$ (not necessarily distinct) such that 
(1) $f_{v_{1}}$ is supported on relatively $\tau$-elliptic elements of $G\left(F_{v_{1}}\right)$,

(2) $f_{v_{2}}$ is supported on strongly relatively $\tau$-regular elements of $G\left(F_{v_{2}}\right)$,

(3) $f_{v_{3}}$ is F-supercuspidal.

Then we have that

$$
\sum_{\{\gamma\}} c^{\tau}(\gamma) \operatorname{TRO}_{\gamma}^{\mu}(f)=\sum_{\pi} \operatorname{TRT}_{G^{\sigma}, G^{\theta}}^{\mu}\left(\pi\left(f^{1}\right)\right),
$$

where the sum on the left is over relevant strongly relatively $\tau$-regular elliptic classes in $G(F)$ and the sum on the right is over equivalence classes of automorphic representations $\pi$ of ${ }^{1} G\left(\mathbb{A}_{F}\right)$ admitting a realization in $L_{0}^{2}\left(G(F) \backslash^{1} G\left(\mathbb{A}_{F}\right)\right.$. Here $f^{1}$ is a normalization of $f$ defined in (4.5) and ${ }^{1} G\left(\mathbb{A}_{F}\right)$ is the Harish-Chandra subgroup of $G\left(\mathbb{A}_{F}\right)($ see $\S 2)$.

Remarks.

(1) In the theorem, $c^{\tau}(\gamma)$ is a volume term, $\operatorname{TRO}_{\gamma}^{\mu}(f)$ is a twisted relative orbital integral as in (3.3), and $\operatorname{TRT}_{G^{\sigma}, G^{\theta}}^{\mu}\left(\pi\left(f^{1}\right)\right)$ is a twisted relative trace as in (5.5).

(2) If $f^{1}$ is $K_{\infty}$-finite, where $K_{\infty}$ is a maximal compact subgroup of $G\left(\mathbb{A}_{F, \infty}\right)$, then

$$
\operatorname{TRT}_{G^{\sigma}, G^{\theta}}^{\mu}\left(\pi\left(f^{1}\right)\right)=\sum_{\phi \in \mathcal{B}_{\pi}} \mathcal{P}_{\mu_{1}}^{\sigma}\left(\pi\left(f^{1}\right) \phi\right) \overline{\mathcal{P}_{\mu_{2}}^{\theta}(\phi)},
$$

where $\mathcal{P}_{\mu_{1}}^{\sigma}(\phi)$ and $\mathcal{P}_{\mu_{2}}^{\theta}(\phi)$ are period integrals of $\phi$ over $G^{\sigma}$ and $G^{\theta}$, respectively, (see (5.1) and (5.2) for definitions). Here $\mathcal{B}_{\pi}$ is an orthonormal basis of the $\pi$-isotypic subspace of $L_{0}^{2}\left(G(F) \backslash{ }^{1} G\left(\mathbb{A}_{F}\right)\right)$ with respect to the pairing (5.3) that consists of smooth $K_{\infty}$-finite vectors.

(3) The condition that $G^{\sigma}$ is connected in the theorem is used to prove Proposition 3.4 and to apply $[\mathrm{AGR}, \S 2$, Proposition 1] to prove Proposition 5.2.

Theorem 1.2 will be restated and proven in $\S 5$. The proof of the theorem roughly follows $[\mathrm{BDKV}]$. However, our proof uses Galois cohomology in place of reduction theory to prove that the geometric side of the twisted relative trace formula is a finite sum.

In [GW], our simple twisted relative trace formula is used to prove the following result: Let $U^{\prime}$ be a quasi-split unitary group of odd rank $n$ with respect to a CM extension $M / F$, let $E / F$ be a totally real quadratic extension with $\langle\sigma\rangle=\operatorname{Gal}(E / F)$, and let $U:=\operatorname{Res}_{E / F} U^{\prime}$. Under suitable local hypotheses, a cuspidal cohomological automorphic representation $\pi$ of $U$ such that $\pi^{\vee} \cong \pi^{\sigma}$ is nearly equivalent to cohomological automorphic representation $\pi^{\prime}$ of $U$ that is $U^{\prime}$-distinguished in the sense that there is a form in the space of $\pi^{\prime}$ admitting a nonzero period over $U^{\prime}$.

\section{PRELiminaries}

2.1. Algebraic groups. Let $G_{0}$ be an algebraic group over a field $F$. We write $Z_{G_{0}}$ for the center of $G_{0}$ and $G_{0}^{\circ}$ for the identity component of $G_{0}$ in the Zariski topology. If $\gamma \in G_{0}(F)$ and $G_{0}^{\prime} \leq G_{0}$ is a subgroup we write $C_{G_{0}^{\prime}, \gamma}$ for the centralizer in $G_{0}^{\prime}$ of $\gamma$.

Let $M / F$ be a quadratic extension of $F$-algebras. Thus $M$ is either a field or $M=$ $F \oplus F$. Let $G:=\operatorname{Res}_{M / F} G_{0}$ and let $\tau$ be the automorphism of $G$ induced by the 
generator of $\operatorname{Gal}(M / F)$. Assume $G_{0}$ (and hence $G$ ) is connected. As usual, an element $\gamma \in G(F)$ is said to be $\tau$-semisimple if $\gamma \gamma^{\tau}$ is semisimple. A $\tau$-semisimple element $\gamma$ is $\tau$-elliptic (resp. $\tau$-regular semisimple, $\tau$-regular elliptic) if $C_{G, \gamma}^{\tau} / Z_{G_{0}}$ is anisotropic (resp. a torus, an anisotropic torus).

If $\sigma$ is an automorphism of $G$ and $\gamma \in G(R)$ for some commutative $F$-algebra $R$ we write

$$
\gamma^{-\sigma}:=\left(\gamma^{-1}\right)^{\sigma}
$$

2.2. Adèles. The adèles of a number field $F$ will be denoted by $\mathbb{A}_{F}$. For a set of places $V$ of $F$ we write $\mathbb{A}_{F, V}:=\otimes_{v \in V}^{\prime} F_{v}$ and $\mathbb{A}_{F}^{V}:=\otimes_{v \notin V}^{\prime} F_{v}$. Here $\otimes^{\prime}$ denotes the typical restricted direct product. The set of infinite places of $F$ will be denoted by $\infty$. For an affine $F$-variety $G$ and a subset $W \leq G\left(\mathbb{A}_{F}\right)$ the notation $W_{V}$ (resp. $W^{V}$ ) will denote the projection of $W$ to $G\left(\mathbb{A}_{F, V}\right)$ (resp. $G\left(\mathbb{A}_{F}^{V}\right)$ ). If $W$ is replaced by an element of $G\left(\mathbb{A}_{F}\right)$, or if $G$ is an algebraic group and $W$ is replaced by a character of $G\left(\mathbb{A}_{F}\right)$ or a Haar measure on $G$, the analogous notation will be in force; for example, if $\gamma \in G\left(\mathbb{A}_{F}\right)$ then $\gamma_{v}$ is the projection to $G\left(F_{v}\right)$ of $\gamma$.

2.3. Harish-Chandra subgroups. Let $G$ be a connected reductive group over a number field $F$. We write $A_{G} \leq Z_{G}\left(F \otimes_{\mathbb{Q}} \mathbb{R}\right)$ for the connected component of the real points of the largest $F$-split torus in the center of $G$. Write $X^{*}$ for the group of $F$-rational characters of $G$ and write $\mathfrak{a}_{G}:=\operatorname{Hom}\left(X^{*}, \mathbb{R}\right)$. The Harish-Chandra morphism

$$
H C_{G}: G\left(\mathbb{A}_{F}\right) \longrightarrow \mathfrak{a}_{G}
$$

is defined by

$$
\left\langle H C_{G}(x), \chi\right\rangle=\left|\log \left(x^{\chi}\right)\right|
$$

for $x \in G\left(\mathbb{A}_{F}\right)$ and $\chi \in X^{*}$. We write

$$
{ }^{1} G\left(\mathbb{A}_{F}\right):=\operatorname{ker}\left(H C_{G}\right)
$$

and refer to it as the Harish-Chandra subgroup. Note that $G\left(\mathbb{A}_{F}\right)$ is the direct product of $A_{G}$ and ${ }^{1} G\left(\mathbb{A}_{F}\right)$, and that $G(F) \leq{ }^{1} G\left(\mathbb{A}_{F}\right)$.

2.4. Definitions and notations. Let $G_{0}$ be a connected reductive $F$-group with automorphism $\sigma$ of order 2 and let $G_{0}^{\sigma}, G$, etc. be defined as in the introduction. We have left actions of $G_{0}^{\sigma} \times G_{0}^{\sigma}$ on $G_{0}$ and $G^{\sigma} \times G^{\theta}$ on $G$ given by

$$
\begin{aligned}
\left(G_{0}^{\sigma} \times G_{0}^{\sigma}\right)(R) \times G_{0}(R) & \longrightarrow G_{0}(R) \\
\left(\left(g_{1}, g_{2}\right), g\right) & \longmapsto g_{1} g g_{2}^{-1}, \\
\left(G^{\sigma} \times G^{\theta}\right)(R) \times G(R) & \longrightarrow G(R) \\
\left(\left(g_{1}, g_{2}\right), g\right) & \longmapsto g_{1} g g_{2}^{-1}
\end{aligned}
$$


for $F$-algebras $R$. Following [Ri, Lemma 2.4] we introduce the subschemes $Q \subset G_{0}$ and $S \subset G$ defined as the scheme theoretic images of the morphisms given on $R$-points by

$$
\begin{aligned}
B_{\sigma}: G_{0}(R) & \longrightarrow G_{0}(R) \\
g & \longmapsto g g^{-\sigma}, \\
B_{\theta}: G(R) & \longrightarrow G(R) \\
g & \longmapsto g g^{-\theta},
\end{aligned}
$$

respectively. Here $R$ is a (commutative) $F$-algebra. The $F$-schemes $Q$ and $S$ are closed affine $F$-subschemes of $G_{0}$ and $G$, respectively (see $[H W, \S 2.1]$ ). Note that

$$
B_{\sigma}\left(\left(g_{1}, g_{2}\right) \cdot g\right)=g_{1} B_{\sigma}(g) g_{1}^{-1} \quad \text { and } \quad B_{\theta}\left(\left(g_{1}, g_{2}\right) \cdot g\right)=g_{1} B_{\theta}(g) g_{1}^{-\tau},
$$

where denotes the left actions defined by (2.2) and (2.3) respectively.

The following definition is an adaptation of one appearing in $[\mathrm{F}]$ and $[\mathrm{JL}]$ (see $[\mathrm{GW}]$ ):

Definition 2.1. Let $k$ be a field extension of $F$. An element $\gamma \in G(k)$ is relatively $\tau$-semisimple (resp. relatively $\tau$-elliptic, relatively $\tau$-regular semisimple, relatively $\tau$ regular elliptic) if $\gamma \gamma^{-\theta}$ is $\tau$-semisimple (resp. $\tau$-elliptic, $\tau$-regular semisimple, $\tau$-regular elliptic) as an element of $G(k)$.

Definition 2.2. For any $F$-algebra $k$ and any $\gamma \in G(k)$, write $G_{\gamma}^{\sigma \theta}$ for the $k$-group whose points in a commutative $k$-algebra $R$ are given by

$$
G_{\gamma}^{\sigma \theta}(R):=\left\{(x, y) \in G^{\sigma}(R) \times G^{\theta}(R): x^{-1} \gamma y=\gamma\right\} .
$$

Write $C_{G^{\sigma}, \gamma}^{\tau}$ for the $\tau$-centralizer of $\gamma$ in $G^{\sigma}$. Explicitly, $C_{G^{\sigma}, \gamma}^{\tau}$ is the $k$-group whose points in a commutative $k$-algebra $R$ are given by

$$
C_{G^{\sigma}, \gamma}^{\tau}(R):=\left\{x \in G^{\sigma}(R): x \gamma x^{-\tau}=\gamma\right\} .
$$

Definition 2.3. Let $k$ be an $F$-algebra. A relatively $\tau$-semisimple element $\gamma \in G(k)$ is strongly relatively $\tau$-regular if $C_{G^{\sigma}, \gamma \gamma^{-\theta}}^{\tau}$ is a (connected) torus.

Thus a strongly relatively $\tau$-regular element is relatively $\tau$-regular semisimple.

For an $F$-algebra $R$, write

$$
\Gamma_{r}(R):=G^{\sigma}(R) \backslash G(R) / G^{\theta}(R) .
$$

We refer to the elements of $\Gamma_{r}(R)$ as relative $\tau$-classes. If two elements $\gamma, \gamma^{\prime} \in G(R)$ map to the same class in $\Gamma_{r}(R)$, we say that they are in the same relative $\tau$-class.

Let $k$ be $F$ or $F_{v}$ for some place $v$ of $F$. Then we say that two relatively $\tau$-semisimple $\gamma, \gamma^{\prime} \in G(k)$ are in the same geometric relative $\tau$-class if there exists $(\bar{x}, \bar{y}) \in$ $G^{\sigma}(\bar{k}) \times G^{\theta}(\bar{k})$ such that $\bar{x}^{-1} \gamma \bar{y}=\gamma^{\prime}$. We write $\Gamma_{r}^{\text {ge }}(k)$ for the set of geometric relative $\tau$-classes. Notice that if $\gamma$ and $\gamma^{\prime}$ are in the same geometric relative $\tau$-class then $\gamma$ is strongly relatively $\tau$-regular if and only if $\gamma^{\prime}$ is as well.

Lemma 2.4. Let $k$ be $F$ or $F_{v}$ for some place $v$ of $F$. If $\gamma \in G(k)$ is relatively $\tau$-semisimple then the $G^{\sigma}$-orbit of $\gamma \gamma^{-\theta}$ in $S_{k}$ is closed. 
Proof. If $\tau$ is trivial, this is Theorem 7.5 of [Ri], so we assume for the remainder of the proof that $\tau$ is nontrivial. Let $\bar{k}$ be an algebraic closure of $k$. By faithfully flat descent along $k \rightarrow \bar{k}$, it is enough to prove that the orbit of $\gamma \gamma^{-\theta}$ under $G_{\bar{k}}^{\sigma}$ is closed. Choose an isomorphism $G_{\bar{k}} \cong G_{0 \bar{k}} \times G_{0 \bar{k}}$ intertwining $\tau$ with $(x, y) \longmapsto(y, x)$ and equivariant with respect to $\sigma$. Note that there is a morphism $S_{\bar{k}} \rightarrow Q_{\bar{k}}$ given at the level of $R$-points by

$$
\begin{gathered}
N: S_{\bar{k}}(R) \longrightarrow Q_{\bar{k}}(R) \\
\left(x_{1}, x_{2}\right) \longmapsto x_{1} x_{2},
\end{gathered}
$$

for $\bar{k}$-algebra $R$. The element $N\left(\gamma \gamma^{-\theta}\right) \in Q(\bar{k})$ is semisimple by assumption, so by Theorem 7.5 of [Ri] we conclude that its $G_{0 \bar{k}}^{\sigma}$ conjugacy class is closed. The inverse image of the $G_{0 \bar{k}}^{\sigma}$ conjugacy class of $N\left(\gamma \gamma^{-\theta}\right)$ is the $G_{\bar{k}}^{\sigma}$ orbit of $\gamma \gamma^{-\theta}$ (under $\tau$-conjugation). The $G_{\bar{k}}^{\sigma}$ orbit of $\gamma \gamma^{-\theta}$ under $\tau$-conjugation is therefore closed since it is the inverse image of a closed set under a continuous map.

Theorem 2.5. If $\gamma \in G(k)$ is relatively $\tau$-semisimple, then $G_{\gamma}^{\sigma \theta}$ is reductive.

Proof. First, we need to show that the morphism from $G_{\gamma}^{\sigma \theta}$ to $C_{G^{\sigma}, \gamma \gamma^{-\theta}}^{\tau}$ given on $R$ points by

$$
\begin{aligned}
G_{\gamma}^{\sigma \theta}(R) & \rightarrow C_{G^{\sigma}, \gamma \gamma^{-\theta}}^{\tau}(R) \\
(x, y) & \mapsto x
\end{aligned}
$$

is an isomorphism. It is clearly injective. For surjectivity, if $x^{-1} \gamma \gamma^{-\theta} x^{\tau}=\gamma \gamma^{-\theta}$ then

$$
\gamma^{-1} x^{-1} \gamma=\gamma^{-\theta} x^{-\tau} \gamma^{\theta}=\left(\gamma^{-1} x^{-\sigma} \gamma\right)^{\theta}=\left(\gamma^{-1} x^{-1} \gamma\right)^{\theta}
$$

so $\left(x,\left(\gamma^{-1} x^{-1} \gamma\right)^{-1}\right) \in G_{\gamma}^{\sigma \theta}(R)$.

Notice that $C_{G^{\sigma}, \gamma \gamma^{-\theta}}^{\tau}$ is the stabilizer of $\gamma \gamma^{-\theta} \in S(k)$ under the action of $G^{\sigma}$ on $S$ by $\tau$-conjugation. Since the $G^{\sigma}$-orbit of $\gamma \gamma^{-\theta}$ is closed, it follows that $C_{G^{\sigma}, \gamma \gamma^{-\theta}}^{\tau}$ is reductive [BR, 2.1.10 (b)].

\section{TWISTED RELATIVE ORBITAL INTEGRALS}

Let $v$ be a place of $F$ and let

$$
\mu_{v}:=\mu_{1 v} \times \mu_{2 v}: G^{\sigma}\left(F_{v}\right) \times G^{\theta}\left(F_{v}\right) \rightarrow \mathbb{C}^{\times}
$$

be a unitary character. Let $\gamma_{v} \in G\left(F_{v}\right)$ be a relatively $\tau$-semisimple element. Since $\left(G_{\gamma_{v}}^{\sigma \theta}\right)^{\circ}$ is reductive, the closed subgroup

$$
\left(G_{\gamma_{v}}^{\sigma \theta}\right)^{\circ}\left(F_{v}\right) \leq G^{\sigma}\left(F_{v}\right) \times G^{\theta}\left(F_{v}\right)
$$

is unimodular. Thus we can and do choose Haar measures $d x_{v}, d y_{v}$, and $d t_{\gamma_{v}}$ on $G^{\sigma}\left(F_{v}\right)$, $G^{\theta}\left(F_{v}\right)$ and $\left(G_{\gamma_{v}}^{\sigma \theta}\right)^{\circ}\left(F_{v}\right)$, respectively, and form the right invariant Radon measure $\frac{d x_{v} d y_{v}}{d t_{\gamma_{v}}}$.

Definition 3.1. Let $\gamma_{v} \in G\left(F_{v}\right)$ be a relatively $\tau$-semisimple element. Then $\gamma_{v}$ is said to be a relevant element if $\mu_{v}$ is trivial on $\left(G_{\gamma_{v}}^{\sigma \theta}\right)^{\circ}\left(F_{v}\right)$. 
For $f_{v} \in C_{c}^{\infty}\left(G\left(F_{v}\right)\right)$ and for a relevant element $\gamma_{v}$, the twisted relative orbital integral is defined by

$$
\operatorname{TRO}_{\gamma_{v}}^{\mu_{v}}\left(f_{v}\right):=\iint_{\left(G_{\left.\gamma_{v}\right)^{\circ}\left(F_{v}\right) \backslash G^{\sigma}\left(F_{v}\right) \times G^{\theta}\left(F_{v}\right)}\right.} \mu_{v}\left(x_{v}, y_{v}^{-1}\right) f_{v}\left(x_{v}^{-1} \gamma_{v} y_{v}\right) \frac{d x_{v} d y_{v}}{d t_{\gamma_{v}}} .
$$

The orbital integral (3.1) is absolutely convergent:

Proposition 3.2. The twisted relative orbital integral defined in (3.1) is absolutely convergent.

Proof. Recall that $\left|\mu_{v}\right|=1$ so it plays no role in absolute convergence. There is a surjective map $[\mathrm{RR}, \S 3]$

$$
\begin{aligned}
C_{c}^{\infty}\left(G\left(F_{v}\right)\right) & \longrightarrow C_{c}^{\infty}\left(S\left(F_{v}\right)\right) \\
f_{v} & \longmapsto \psi_{f_{v}}
\end{aligned}
$$

where

$$
\psi_{f_{v}}\left(g_{v} g_{v}^{-\theta}\right):=\int_{G^{\theta}\left(F_{v}\right)} f_{v}\left(g_{v} y_{v}\right) d y_{v}, \quad g_{v} \in G\left(F_{v}\right) .
$$

Note that it is not necessarily true that all of the elements of $S\left(F_{v}\right)$ are of the form $g_{v} g_{v}^{-\theta}$ for $g_{v} \in G\left(F_{v}\right)$. We have that

$$
\begin{aligned}
\iint_{\left(G_{\gamma v}^{\sigma \theta}\right)^{\circ}\left(F_{v}\right) \backslash G^{\sigma}\left(F_{v}\right) \times G^{\theta}\left(F_{v}\right)} & \left|f_{v}\left(x_{v}^{-1} \gamma_{v} y_{v}\right)\right| \frac{d x_{v} d y_{v}}{d t_{\gamma_{v}}} \\
= & \int_{\left(C_{G^{\sigma}, \gamma_{v} \gamma_{v}^{-\theta}}^{\tau}\right)^{\circ}\left(F_{v}\right) \backslash G^{\sigma}\left(F_{v}\right)}\left|\psi_{f_{v}}\left(g_{v}\left(\gamma_{v} \gamma_{v}^{-\theta}\right) g_{v}^{-\tau}\right)\right| d g_{v} \\
= & \int_{\left(C_{G^{\sigma}, \gamma_{v} \gamma_{v}^{-\theta}}^{\tau}\right)^{\circ}\left(F_{v}\right) \backslash G^{\sigma}\left(F_{v}\right)}\left|\psi_{f_{v}}\left(g_{v} \gamma_{v}\left(g_{v} \gamma_{v}\right)^{-\theta}\right)\right| d g_{v} .
\end{aligned}
$$

The $G^{\sigma}\left(F_{v}\right)$-orbit of $\gamma_{v} \gamma_{v}^{-\theta}$ in $S\left(F_{v}\right)$ is closed by Lemma 2.4. It follows that the support of the function

$$
\begin{aligned}
\left(C_{G^{\sigma}, \gamma_{v} \gamma_{v}^{-\theta}}^{\tau}\right)^{\circ}\left(F_{v}\right) \backslash G^{\sigma}\left(F_{v}\right) & \longrightarrow \mathbb{C} \\
g_{v} & \longmapsto \psi_{f_{v}}\left(g_{v}\left(\gamma_{v} \gamma_{v}^{-\theta}\right) g_{v}^{-\tau}\right)
\end{aligned}
$$

is compact, so the convergence of the right hand side of (3.2) is immediate.

Let

$$
\begin{aligned}
& \mu_{1}: G^{\sigma}\left(\mathbb{A}_{F}\right) \longrightarrow \mathbb{C}^{\times}, \\
& \mu_{2}: G^{\theta}\left(\mathbb{A}_{F}\right) \longrightarrow \mathbb{C}^{\times}
\end{aligned}
$$

be unitary characters trivial on $G^{\sigma}(F)$ and $G^{\theta}(F)$, respectively, and set

$$
\mu:=\mu_{1} \times \mu_{2}: G^{\sigma}\left(\mathbb{A}_{F}\right) \times G^{\theta}\left(\mathbb{A}_{F}\right) \longrightarrow \mathbb{C}^{\times} .
$$

Definition 3.3. A relatively $\tau$-semisimple element $\gamma \in G\left(\mathbb{A}_{F}\right)$ is said to be a relevant element if $\mu$ is trivial on $\left(G_{\gamma}^{\sigma \theta}\right)^{\circ}\left(\mathbb{A}_{F}\right)$. 
For $f \in C_{c}^{\infty}\left(\mathbb{A}_{F}\right)$ and for a relevant element $\gamma$, we define

$$
\operatorname{TRO}_{\gamma}^{\mu}(f):=\iint_{\left(G_{\gamma}^{\sigma \theta}\right)^{\circ}\left(\mathbb{A}_{F}\right) \backslash G^{\sigma}\left(\mathbb{A}_{F}\right) \times G^{\theta}\left(\mathbb{A}_{F}\right)} \mu\left(x, y^{-1}\right) f\left(x^{-1} \gamma y\right) \frac{d x d y}{d t_{\gamma}},
$$

where $d x, d y$, and $d t_{\gamma}$ are Haar measures on $G^{\sigma}\left(\mathbb{A}_{F}\right), G^{\theta}\left(\mathbb{A}_{F}\right)$ and $\left(G_{\gamma}^{\sigma \theta}\right)^{\circ}\left(\mathbb{A}_{F}\right)$, respectively. When $G^{\sigma}$ is connected and $\gamma$ is strongly relatively $\tau$-regular, Proposition 3.2 and Proposition 3.4 below imply that (3.3) is absolutely convergent.

Proposition 3.4. Suppose that $G^{\sigma}$ is connected and $\gamma \in G(F)$ is a relevant strongly relatively $\tau$-regular element. Then, for almost all finite places $v$, we have that $G^{\sigma}, G^{\theta}$, and $G$ are unramified at $v$ and we have that

$$
\operatorname{TRO}_{\gamma_{v}}^{\mu_{v}}\left(\operatorname{ch}_{K_{v}}\right)=\iint_{G_{\gamma_{v}}^{\sigma \theta}\left(F_{v}\right) \cap\left(K_{v}^{\sigma} \times K_{v}^{\theta}\right) \backslash K_{v}^{\sigma} \times K_{v}^{\theta}} \frac{d x_{v} d y_{v}}{d t_{\gamma_{v}}}=1,
$$

where $K_{v} \leq G\left(F_{v}\right)$ is a hyperspecial subgroup such that

$$
K_{v}^{\sigma}:=K_{v} \cap G^{\sigma}\left(F_{v}\right), \quad K_{v}^{\theta}:=K_{v} \cap G^{\theta}\left(F_{v}\right),
$$

and

$$
G_{\gamma_{v}}^{\sigma \theta}\left(F_{v}\right) \cap\left(K_{v}^{\sigma} \times K_{v}^{\theta}\right) \leq G_{\gamma_{v}}^{\sigma \theta}\left(F_{v}\right)
$$

are all hyperspecial. Here $d x_{v}, d y_{v}$, and $d t_{\gamma_{v}}$ are normalized to give $K_{v}^{\sigma}, K_{v}^{\theta}$ and $G_{\gamma_{v}}^{\sigma \theta}\left(F_{v}\right) \cap\left(K_{v}^{\sigma} \times K_{v}^{\theta}\right)$ volume 1 , respectively. As usual, $\mathrm{ch}_{K_{v}}$ is the characteristic function of $K_{v}$.

Proof. Let $\gamma \in G(F)$ be strongly relatively $\tau$-regular and let $O(\gamma)$ denote its geometric relative $\tau$-class. We view $O(\gamma)$ as an affine $F$-subscheme of $G$. Note that the categorical quotient $G_{\gamma}^{\sigma \theta} \backslash\left(G^{\sigma} \times G^{\theta}\right)$ exists as an affine scheme of finite type over $F$ (see [GIT, Theorem $1.1 \S 1.2])$. There is a natural isomorphism

$$
G_{\gamma}^{\sigma \theta} \backslash\left(G^{\sigma} \times G^{\theta}\right) \cong O(\gamma)
$$

induced by the morphism $G^{\sigma} \times G^{\theta} \longrightarrow G$ given on $F$-algebras $R$ by

$$
\begin{aligned}
G^{\sigma}(R) \times G^{\theta}(R) & \longrightarrow G(R) \\
(x, y) & \longmapsto x \gamma y^{-1} .
\end{aligned}
$$

Choose an embedding $G \longrightarrow \mathrm{GL}_{n}(F)$ for some integer $n$ and let $\mathcal{G}$ be the scheme theoretic closure of $G$ in $\mathrm{GL}_{n}\left(\mathcal{O}_{F}\right)$. Moreover, let

$$
\mathcal{O}(\gamma), \mathcal{G}^{\sigma}, \mathcal{G}^{\theta} \subset \mathcal{G} \text { and } \mathcal{G}_{\gamma}^{\sigma \theta} \subset \mathcal{G}^{\sigma} \times \mathcal{G}^{\theta}
$$

be the scheme theoretic closures of $O(\gamma), G^{\sigma}, G^{\theta}$ and $G_{\gamma}^{\sigma \theta}$, respectively. Then, for almost all finite places $v$ of $F$, the group schemes $\mathcal{G}^{\sigma}, \mathcal{G}^{\theta}, \mathcal{G}$ and $\mathcal{G}_{\gamma}^{\sigma \theta}$ are reductive and their $\mathcal{O}_{F_{v}}$-points are hyperspecial subgroups of their $F_{v}$-points $[\mathrm{T}, \S 3.9]$. Here we are using the fact that $\gamma$ is strongly relatively $\tau$-regular to conclude that $G_{\gamma}^{\sigma \theta}$ is connected.

Moreover, there is an integer $N$ such that the natural morphism

$$
\left(\mathcal{G}^{\sigma} \times \mathcal{G}^{\theta}\right)_{\mathcal{O}_{F}\left[N^{-1}\right]} \longrightarrow \mathcal{O}(\gamma)_{\mathcal{O}_{F}\left[N^{-1}\right]}
$$

is a smooth morphism between smooth schemes whose generic fiber is induced by (3.5). 
Now let $v$ be a finite place of $F$ whose residual characteristic is prime to $N$. By enlarging $N$ if necessary, we can and do assume that $\gamma \in \mathcal{G}\left(\mathcal{O}_{F_{v}}\right)$. We claim that the map

$$
\mathcal{G}^{\sigma}\left(\mathcal{O}_{F_{v}}\right) \times \mathcal{G}^{\theta}\left(\mathcal{O}_{F_{v}}\right) \longrightarrow \mathcal{O}(\gamma)\left(\mathcal{O}_{F_{v}}\right)
$$

is surjective. Indeed, let $\gamma^{\prime} \in \mathcal{O}(\gamma)\left(\mathcal{O}_{F_{v}}\right)$, let $\mathbb{F}_{v}$ be the residue field of $\mathcal{O}_{F_{v}}$, and let $\bar{\gamma}^{\prime} \in \mathcal{O}(\gamma)\left(\mathbb{F}_{v}\right)$ be the image of $\gamma^{\prime}$ under the reduction map. Consider the set of $(\bar{x}, \bar{y}) \in \mathcal{G}^{\sigma}\left(\mathbb{F}_{v}\right) \times \mathcal{G}^{\theta}\left(\mathbb{F}_{v}\right)$ such that $\overline{x \gamma}^{\prime} \bar{y}^{-1}=\bar{\gamma}$. This is a (nonempty) $\mathcal{G}_{\gamma \mathbb{F}_{v}}^{\sigma \theta}$-homogeneous space. Since $\gamma$ is strongly relatively $\tau$-regular, $\mathcal{G}_{\gamma \mathbb{F}_{v}}^{\sigma \theta}$ is connected and hence has an $\mathbb{F}_{v^{-}}$ point [S1, Theorem 1.9]. In other words, there is a $(x, y) \in \mathcal{G}^{\sigma}\left(\mathbb{F}_{v}\right) \times \mathcal{G}^{\theta}\left(\mathbb{F}_{v}\right)$ such that $x \bar{\gamma}^{\prime} y^{-1}=\bar{\gamma}$. Since $\mathcal{G}^{\sigma} \times \mathcal{G}^{\theta}$ is smooth, this implies that there is a lift of $(x, y)$ to an element of $\mathcal{G}^{\sigma}\left(\mathcal{O}_{F_{v}}\right) \times \mathcal{G}^{\theta}\left(\mathcal{O}_{F_{v}}\right)$ [BLR, Proposition 5].

Summing up, if the residual characteristic of a finite place $v$ of $F$ is large enough and $\gamma$ is an element of the hyperspecial subgroup $K:=\mathcal{G}\left(\mathcal{O}_{F_{v}}\right)$, then any $\gamma^{\prime} \in K$ in the geometric relative $\tau$-class of $\gamma$ in fact satisfies $\gamma^{\prime} \in K^{\sigma} \gamma K^{\theta}$, where $K^{\sigma}:=\mathcal{G}^{\sigma}\left(\mathcal{O}_{F_{v}}\right)$ and $K^{\theta}:=\mathcal{G}^{\theta}\left(\mathcal{O}_{F_{v}}\right)$.

Thus, for almost all $v$ we have that $\mathrm{TRO}_{\gamma_{v}}^{\mu_{v}}\left(\mathrm{ch}_{K_{v}}\right)$ is equal to

$$
\iint_{G_{\gamma_{v}}^{\sigma \theta}\left(F_{v}\right) \cap\left(K_{v}^{\sigma} \times K_{v}^{\theta}\right) \backslash\left(K_{v}^{\sigma} \times K_{v}^{\theta}\right)} \mu_{v}\left(x_{v}, y_{v}^{-1}\right) \frac{d x_{v} d y_{v}}{d t_{\gamma_{v}}} .
$$

The character $\mu_{v}$ is trivial on $K_{v}$ for almost all $v$, which completes the proof.

\section{Geometric expansion for the elliptic Kernel}

In this section, we provide a geometric expansion of the relatively $\tau$-elliptic portions of the twisted relative trace formula. Throughout this section and for the rest of the paper, we assume that $G^{\sigma}$ is connected.

Recall the central subgroup $A_{G}$ and the Harish-Chandra subgroup ${ }^{1} G\left(\mathbb{A}_{F}\right)$ defined in (2.1). To ease notation, set

$$
{ }^{2} G^{\sigma}\left(\mathbb{A}_{F}\right):=G^{\sigma}\left(\mathbb{A}_{F}\right) \cap{ }^{1} G\left(\mathbb{A}_{F}\right), \quad{ }^{2} G^{\theta}\left(\mathbb{A}_{F}\right):=G^{\theta}\left(\mathbb{A}_{F}\right) \cap{ }^{1} G\left(\mathbb{A}_{F}\right)
$$

and

$$
A_{G}^{\sigma}:=A_{G} \cap G^{\sigma}\left(F \otimes_{\mathbb{Q}} \mathbb{R}\right), \quad A_{G}^{\theta}:=A_{G} \cap G^{\theta}\left(F \otimes_{\mathbb{Q}} \mathbb{R}\right) .
$$

Define

$$
A:=\left\{(z, z) \in A_{G}^{\sigma} \times A_{G}^{\theta}: z \in A_{G}^{\sigma} \cap A_{G}^{\theta}\right\} .
$$

For every $\gamma \in G(F)$, define

$$
{ }^{2} G_{\gamma}^{\sigma \theta}\left(\mathbb{A}_{F}\right):=\left(G_{\gamma}^{\sigma \theta}\right)^{\circ}\left(\mathbb{A}_{F}\right) \cap\left({ }^{2} G^{\sigma}\left(\mathbb{A}_{F}\right) \times{ }^{2} G^{\theta}\left(\mathbb{A}_{F}\right)\right) .
$$

Then we decompose

$$
\left(G_{\gamma}^{\sigma \theta}\right)^{\circ}\left(\mathbb{A}_{F}\right) \backslash\left(G^{\sigma} \times G^{\theta}\right)\left(\mathbb{A}_{F}\right) \cong\left(A \backslash A_{G}^{\sigma} \times A_{G}^{\theta}\right) \times\left({ }^{2} G_{\gamma}^{\sigma \theta}\left(\mathbb{A}_{F}\right) \backslash^{2} G^{\sigma}\left(\mathbb{A}_{F}\right) \times{ }^{2} G^{\theta}\left(\mathbb{A}_{F}\right)\right) .
$$

We now specify a choice of Haar measure on all of the groups appearing in (4.4), at least if $\gamma$ is relatively $\tau$-elliptic. Whenever a reductive $F$-group $H$ appears, we give $H\left(\mathbb{A}_{F}\right), A_{H},{ }^{1} H\left(\mathbb{A}_{F}\right)$ the measures that are used in the definition of the Tamagawa 
number $\tau(H)=\left[H: H^{\circ}\right] \tau\left(H^{\circ}\right)$. We then fix, once and for all, measures $d z_{\sigma}$ for $A_{G}^{\sigma}$, $d z_{\theta}$ for $A_{G}^{\theta}$, and stipulate that the isomorphisms

$$
\begin{aligned}
{ }^{2} G^{\sigma}\left(\mathbb{A}_{F}\right) & \cong A_{G^{\sigma}} / A_{G}^{\sigma} \times{ }^{1} G^{\sigma}\left(\mathbb{A}_{F}\right) \\
{ }^{2} G^{\theta}\left(\mathbb{A}_{F}\right) & \cong A_{G^{\theta}} / A_{G}^{\theta} \times{ }^{1} G^{\theta}\left(\mathbb{A}_{F}\right)
\end{aligned}
$$

are measure preserving. Notice that if $\gamma$ is relatively $\tau$-elliptic then

$$
{ }^{2} G_{\gamma}^{\sigma \theta}\left(\mathbb{A}_{F}\right)={ }^{1}\left(G_{\gamma}^{\sigma \theta}\right)^{\circ}\left(\mathbb{A}_{F}\right)
$$

By our earlier convention, we have already endowed ${ }^{2} G_{\gamma}^{\sigma \theta}\left(\mathbb{A}_{F}\right)$ and $A=A_{G_{\gamma}^{\sigma \theta}}$ with Haar measures. Let $d t$ be this Haar measure for $A$. Altogether, this endows all of the groups occurring in (4.4) with measures. Thus ${ }^{2} G_{\gamma}^{\sigma \theta}\left(\mathbb{A}_{F}\right)$ is given the unique Haar measure such that

$$
\operatorname{vol}\left(\left(G_{\gamma}^{\sigma \theta}\right)^{\circ}(F) \backslash^{2} G_{\gamma}^{\sigma \theta}\left(\mathbb{A}_{F}\right)\right)=\tau\left(\left(G_{\gamma}^{\sigma \theta}\right)^{\circ}\right) .
$$

For the rest of this paper, when we form twisted relative orbital integrals we use these choices of measures.

For $f$ in $C_{c}^{\infty}\left(G\left(\mathbb{A}_{F}\right)\right)$, define

$$
f^{1}(x)=\int_{A \backslash\left(A_{G}^{\sigma} \times A_{G}^{\theta}\right)} f\left(z_{\sigma}^{-1} z_{\theta} x\right) \frac{d z_{\sigma} d z_{\theta}}{d t} .
$$

Note that $f^{1} \in C_{c}^{\infty}\left({ }^{1} G\left(\mathbb{A}_{F}\right)\right)$. Then the elliptic kernel function is given by

$$
K_{f^{1}}^{e}(x, y)=\sum_{\gamma \in G(F)} f^{1}\left(x^{-1} \gamma y\right)
$$

where the sum is over relatively $\tau$-regular elliptic $\gamma \in G(F)$. We can now state the geometric expansion of the relatively $\tau$-elliptic portion of the twisted relative trace formula.

Theorem 4.1. Suppose that $G^{\sigma}$ is connected. Let $f=\otimes f_{v} \in C_{c}^{\infty}\left(G\left(\mathbb{A}_{F}\right)\right)$ and define $f^{1}$ as in (4.5). Suppose that there exist places $v_{1}, v_{2}$ of $F$ (not necessarily distinct) such that $f_{v_{1}}$ is supported on relatively $\tau$-elliptic elements and $f_{v_{2}}$ is supported on strongly relatively $\tau$-regular elements. Then we have that

$$
\sum_{\{\gamma\}} c^{\tau}(\gamma) \operatorname{TRO}_{\gamma}^{\mu}(f)=\iint_{G^{\sigma}(F) \times G^{\theta}(F) \backslash^{2} G^{\sigma}\left(\mathbb{A}_{F}\right) \times^{2} G^{\theta}\left(\mathbb{A}_{F}\right)} \mu\left(x, y^{-1}\right) K_{f^{1}}^{e}(x, y) d x d y,
$$

where the sum is over the relevant strongly relatively $\tau$-regular elliptic classes in $G(F)$. Here

$$
c^{\tau}(\gamma):=\operatorname{vol}\left(G_{\gamma}^{\sigma \theta}(F) \backslash^{2} G_{\gamma}^{\sigma \theta}\left(\mathbb{A}_{F}\right)\right),
$$

and ${ }^{2} G^{\sigma}\left(\mathbb{A}_{F}\right),{ }^{2} G^{\theta}\left(\mathbb{A}_{F}\right)$ and ${ }^{2} G_{\gamma}^{\sigma \theta}\left(\mathbb{A}_{F}\right)$ are defined as in (4.1) and (4.3).

Remark. In the theorem, we allow $v_{1}=v_{2}$. It follows from the proof of Theorem 4.1 that the sum of the left is finite, and hence absolutely convergent, and the integral on the right is absolutely integrable.

In order to prove Theorem 4.1, we require the following result: 
Proposition 4.2. Suppose that $G^{\sigma}$ is connected. Let $C \subset G\left(\mathbb{A}_{F}\right)$ be a compact subset such that $C_{v}$ is a hyperspecial subgroup of $G\left(F_{v}\right)$ for almost all finite places $v$ of $F$. There are only finitely many $G^{\sigma}(F) \gamma G^{\theta}(F) \in \Gamma_{r}(F)$ with $\gamma$ strongly relatively $\tau$-regular such that

$$
G^{\sigma}\left(\mathbb{A}_{F}\right) \gamma G^{\theta}\left(\mathbb{A}_{F}\right) \cap C \neq \emptyset .
$$

Assuming Proposition 4.2 for the moment, we now prove Theorem 4.1.

Proof of Theorem 4.1. Suppose that $\gamma$ is a relevant strongly relatively $\tau$-regular element. By Proposition 3.4, we have that

$$
\operatorname{TRO}_{\gamma}^{\mu}(f)=\prod_{v} \operatorname{TRO}_{\gamma_{v}}^{\mu_{v}}\left(f_{v}\right)<\infty .
$$

Note that we have used the assumption that $G^{\sigma}$ is connected in order to apply Proposition 3.4. Clearly $c^{\tau}(\gamma)<\infty$ by the relative $\tau$-ellipticity of $\gamma$. Letting $C$ be the closure of $\operatorname{supp}(f)$ in Proposition 4.2, we see that

$$
\sum_{G^{\sigma}(F) \gamma G^{\theta}(F) \in \Gamma_{r}(F)}\left|c^{\tau}(\gamma) \operatorname{TRO}_{\gamma}^{\mu}(f)\right|<\infty .
$$

Indeed, there are only finitely many nonzero summands. Therefore we derive that

$$
\begin{aligned}
& \sum_{\{\gamma\}} c^{\tau}(\gamma) \operatorname{TRO}_{\gamma}^{\mu}(f) \\
& =\sum_{\{\gamma\}} c^{\tau}(\gamma) \iint_{G_{\gamma}^{\sigma \theta}\left(\mathbb{A}_{F}\right) \backslash G^{\sigma}\left(\mathbb{A}_{F}\right) \times G^{\theta}\left(\mathbb{A}_{F}\right)} \mu\left(x, y^{-1}\right) f\left(x^{-1} \gamma y\right) \frac{d x d y}{d t_{\gamma}} \\
& =\sum_{\{\gamma\}} c^{\tau}(\gamma) \iint_{{ }^{2} G_{\gamma}^{\sigma \theta}\left(\mathbb{A}_{F}\right) \backslash^{2} G^{\sigma}\left(\mathbb{A}_{F}\right) \times{ }^{2} G^{\theta}\left(\mathbb{A}_{F}\right)} \mu\left(x, y^{-1}\right) f^{1}\left(x^{-1} \gamma y\right) d \dot{x} d \dot{y} .
\end{aligned}
$$

Here $d \dot{x} d \dot{y}$ is the appropriate quotient measure, and the sums are over the relevant relatively $\tau$-regular elliptic semisimple classes. Notice that we have used our assumption that $\gamma$ is strongly relatively $\tau$-regular to conclude that $\left(G_{\gamma}^{\sigma \theta}\right)^{\circ}=G_{\gamma}^{\sigma \theta}$. Also in the last equality of (4.8) we have used (4.4) and (4.5).

Notice that for irrelevant $\gamma$ the integral

$$
\iint_{G_{\gamma}^{\sigma \theta}(F) \backslash^{2} G^{\sigma}\left(\mathbb{A}_{F}\right) \times{ }^{2} G^{\theta}\left(\mathbb{A}_{F}\right)} \mu\left(x, y^{-1}\right) f^{1}\left(x^{-1} \gamma y\right) d \dot{x} d \dot{y}=0
$$

since it factors through

$$
\int_{G_{\gamma}^{\sigma \theta}(F) \backslash G_{\gamma}^{\sigma \theta}\left(\mathbb{A}_{F}\right)} \mu\left(g_{1}, g_{2}^{-1}\right) d g_{1} d g_{2}
$$

for appropriate measures $d g_{1}$ and $d g_{2}$. With this in mind, by the definition of the volume term $c^{\tau}(\gamma)$ in (4.7), the last line in (4.8) is equal to

$$
\sum_{\{\gamma\}} \iint_{G_{\gamma}^{\sigma \theta}(F) \backslash^{2} G^{\sigma}\left(\mathbb{A}_{F}\right) \times{ }^{2} G^{\theta}\left(\mathbb{A}_{F}\right)} \mu\left(x, y^{-1}\right) f^{1}\left(x^{-1} \gamma y\right) d \dot{x} d \dot{y}
$$




$$
\begin{aligned}
& =\iint_{G^{\sigma}(F) \times G^{\theta}(F) \backslash{ }^{2} G^{\sigma}\left(\mathbb{A}_{F}\right) \times{ }^{2} G^{\theta}\left(\mathbb{A}_{F}\right)} \mu\left(x, y^{-1}\right) \sum_{\gamma \in G(F)} f^{1}\left(x^{-1} \gamma y\right) d x d y \\
& =\iint_{G^{\sigma}(F) \times G^{\theta}(F) \backslash^{2} G^{\sigma}\left(\mathbb{A}_{F}\right) \times{ }^{2} G^{\theta}\left(\mathbb{A}_{F}\right)} \mu\left(x, y^{-1}\right) K_{f^{1}}^{e}(x, y) d x d y .
\end{aligned}
$$

Here the sum in the second line is over strongly relatively $\tau$-regular elliptic elements by our assumptions on the support of $f$.

We now need to prove Proposition 4.2.

Proof of Proposition 4.2. Let $S$ be the subscheme of $G$ defined as in (2.5). We let $G^{\theta}$ act on $G$ by right multiplication, thus the map

$$
G \stackrel{B_{\theta}}{\longrightarrow} S
$$

is constant on $G^{\theta}$-orbits. Moreover, $B_{\theta}$ intertwines the action of $G^{\sigma}$ on $G$ by left multiplication with the action of $G^{\sigma}$ on $S$ by $\tau$-conjugation. Write

$$
G^{\sigma} \backslash S=\operatorname{Spec}\left(\Gamma\left(S, \mathcal{O}_{S}\right)^{G^{\sigma}}\right)
$$

Let $P: S \rightarrow G^{\sigma} \backslash S$ be the quotient map and consider the composite map

$$
G\left(\mathbb{A}_{F}\right) \stackrel{B_{\theta}}{\longrightarrow} S\left(\mathbb{A}_{F}\right) \stackrel{P}{\longrightarrow}\left(G^{\sigma} \backslash S\right)\left(\mathbb{A}_{F}\right) .
$$

Observe that

$$
\left(P \circ B_{\theta}\right)(C) \cap\left(G^{\sigma} \backslash S\right)(F)
$$

is finite since $\left(G^{\sigma} \backslash S\right)(F)$ is discrete in $\left(G^{\sigma} \backslash S\right)\left(\mathbb{A}_{F}\right)$ if we give the later set the canonical adelic topology. By Lemma 2.4, if $\gamma$ is a relatively $\tau$-semisimple element, then the $G^{\sigma}$ orbit of $\gamma \gamma^{-\theta}$ is a zariski closed subset of $S$. Thus if $\gamma, \gamma^{\prime} \in G(F)$ are relatively $\tau$ semisimple and have the same image in $\left(G^{\sigma} \backslash S\right)\left(\mathbb{A}_{F}\right)$, then

$$
g^{-1} \gamma \gamma^{-\theta} g^{\tau}=\gamma^{\prime} \gamma^{-\theta}
$$

for some $g \in G^{\sigma}(\bar{F})$ (see [GIT, $\left.\S 0.2\right]$ ). Since (4.9) is finite, it follows that there are only finitely many geometric relative $\tau$-classes in $\Gamma_{r}^{g e}(F)$ consisting of relatively $\tau$-semisimple elements that intersect $C$.

Thus to complete the proof of Proposition 4.2, it suffices to show that there are only finitely many relative $\tau$-classes that intersect $C$ and are in a fixed geometric relative $\tau$-class consisting of strongly relatively $\tau$-regular elements. Let $G^{\sigma}(F) \gamma G^{\theta}(F)$ be a relative $\tau$-class with $\gamma \in G(F)$ strongly relatively $\tau$-regular. From the proof of Proposition 3.4, there is a finite set of places $V$ of $F$ such that if $v \notin V$ then $\gamma_{v} \in C_{v}$ and if $\gamma_{v}^{\prime} \in C_{v}$ is in the geometric relative $\tau$-class of $\gamma_{v}$, then

$$
G^{\sigma}\left(F_{v}\right) \gamma_{v} G^{\theta}\left(F_{v}\right)=G^{\sigma}\left(F_{v}\right) \gamma_{v}^{\prime} G^{\theta}\left(F_{v}\right) .
$$

On the other hand, for $v \in V$, a standard Galois cohomology argument [L, §1.8] implies that there are only finitely many elements of $\Gamma_{r}\left(F_{v}\right)$ in a given geometric relative $\tau$-class in $\Gamma_{r}^{\mathrm{ge}}\left(F_{v}\right)$. In summary, there are only finitely many

$$
G^{\sigma}\left(\mathbb{A}_{F}\right) \gamma^{\prime} G^{\theta}\left(\mathbb{A}_{F}\right) \in \Gamma_{r}\left(\mathbb{A}_{F}\right)
$$


that intersect $C$ such that $\gamma_{v} \in G^{\sigma}\left(\bar{F}_{v}\right) \gamma_{v}^{\prime} G^{\theta}\left(\bar{F}_{v}\right)$ for all places $v$. By another standard Galois cohomology argument, the set of elements in $\Gamma_{r}(F)$ contained in a given element of $\Gamma_{r}\left(\mathbb{A}_{F}\right)$ injects into the cohomology group $\mathfrak{E}\left(G_{\gamma}^{\sigma \theta}, G^{\sigma} \times G^{\theta} ; \mathbb{A}_{F} / F\right)$ (compare [L, Lemme 1.8.5]). For the definition of $\left.\mathfrak{E}\left(G_{\gamma}^{\sigma \theta}, G^{\sigma} \times G^{\theta} ; \mathbb{A}_{F} / F\right)\right)$, see $[\mathrm{L}, \S 1.8]$. The group $\mathfrak{E}\left(G_{\gamma}^{\sigma \theta}, G^{\sigma} \times G^{\theta} ; \mathbb{A}_{F} / F\right)$ is finite by [L, Proposition 1.8.4]. This completes the proof.

\section{SPECTRAL EXPANSION}

Recall that

$$
\mu:=\mu_{1} \times \mu_{2}: G^{\sigma}\left(\mathbb{A}_{F}\right) \times G^{\theta}\left(\mathbb{A}_{F}\right) \longrightarrow \mathbb{C}^{\times},
$$

where $\mu_{1}$ and $\mu_{2}$ are unitary characters trivial on $G^{\sigma}(F)$ and $G^{\theta}(F)$, respectively. Let $L_{0}^{2}\left(G(F) \backslash^{1} G\left(\mathbb{A}_{F}\right)\right)$ be the subspace of cusp forms in $L^{2}\left(G(F) \backslash^{1} G\left(\mathbb{A}_{F}\right)\right)$. Let $\pi$ be an automorphic representation of $G\left(\mathbb{A}_{F}\right)$ with unitary central character whose restriction to ${ }^{1} G\left(\mathbb{A}_{F}\right)$ admits a realization in $L_{0}^{2}\left(G(F) \backslash{ }^{1} G\left(\mathbb{A}_{F}\right)\right)$. For smooth $\phi$ in the $\pi$-isotypic subspace of $L_{0}^{2}\left(G(F) \backslash{ }^{1} G\left(\mathbb{A}_{F}\right)\right)$, the $\left(G^{\sigma}, \mu_{1}\right)$ and $\left(G^{\theta}, \mu_{2}\right)$-period integrals are defined by

$$
\begin{aligned}
& \mathcal{P}_{\mu_{1}}^{\sigma}(\phi):=\int_{G^{\sigma}(F) \backslash^{2} G^{\sigma}\left(\mathbb{A}_{F}\right)} \mu_{1}(x) \phi(x) d x, \\
& \mathcal{P}_{\mu_{2}}^{\theta}(\phi):=\int_{G^{\theta}(F) \backslash^{2} G^{\theta}\left(\mathbb{A}_{F}\right)} \mu_{2}(y) \phi(y) d y .
\end{aligned}
$$

Here

$$
{ }^{2} G^{\sigma}\left(\mathbb{A}_{F}\right):=G^{\sigma}\left(\mathbb{A}_{F}\right) \cap{ }^{1} G\left(\mathbb{A}_{F}\right) \text { and } \quad{ }^{2} G^{\theta}\left(\mathbb{A}_{F}\right):=G^{\theta}\left(\mathbb{A}_{F}\right) \cap{ }^{1} G\left(\mathbb{A}_{F}\right)
$$

and $d x$ (resp. $d y$ ) is the choice of Haar measure on $G^{\sigma}(F) \backslash^{2} G^{\sigma}\left(\mathbb{A}_{F}\right)$ (resp. $\left.G^{\theta}(F) \backslash^{2} G^{\theta}\left(\mathbb{A}_{F}\right)\right)$ given in (4.1). Both (5.1) and (5.2) converge absolutely [AGR, §2 Proposition 1].

We now derive a spectral expansion of the integral appearing in Theorem 4.1. We follow Gelbart $[\mathrm{G}, \S 1.2]$ in our exposition. Let $R$ be the regular representation of ${ }^{1} G\left(\mathbb{A}_{F}\right)$ on $L^{2}\left(G(F) \backslash{ }^{1} G\left(\mathbb{A}_{F}\right)\right)$. Since $G(F) \backslash{ }^{1} G\left(\mathbb{A}_{F}\right)$ is not in general compact, $R$ does not decompose discretely. We write $R_{0}$ for the subrepresentation of $R$ on the invariant subspace $L_{0}^{2}\left(G(F) \backslash{ }^{1} G\left(\mathbb{A}_{F}\right)\right) \leq L^{2}\left(G(F) \backslash{ }^{1} G\left(\mathbb{A}_{F}\right)\right)$. Then $R_{0}$ decomposes discretely. We make the following:

Convention 5.1. An automorphic representation of ${ }^{1} G\left(\mathbb{A}_{F}\right)$ is the restriction to ${ }^{1} G\left(\mathbb{A}_{F}\right)$ of an automorphic representation of $G\left(\mathbb{A}_{F}\right)$. Two such representations are equivalent if they are equivalent as representations of ${ }^{1} G\left(\mathbb{A}_{F}\right)$.

Note that, with this convention, an automorphic representation $\pi$ of ${ }^{1} G\left(\mathbb{A}_{F}\right)$ admitting a realization in $R_{0}$ is unitary and hence has unitary central character.

For $f \in C_{c}^{\infty}\left(G\left(\mathbb{A}_{F}\right)\right)$, let $f^{1}$ be defined as in (4.5) and we write $R_{0}(f)=\sum_{\pi} m_{\pi} \pi\left(f^{1}\right)$ where the sum is over equivalence classes of irreducible subrepresentations of $R_{0}$ and $m_{\pi}$ is the mutiplicity of $\pi$ in $R_{0}$. Equivalently $L_{0}^{2}\left(G(F) \backslash{ }^{1} G\left(\mathbb{A}_{F}\right)\right)=\sum V_{\pi}$ where $V_{\pi} \leq L_{0}^{2}\left(G(F) \backslash{ }^{1} G\left(\mathbb{A}_{F}\right)\right)$ is an invariant subspace realizing the $m_{\pi}$ copies of $\pi$. Let $\mathcal{B}_{\pi}$ be an orthonormal basis of $V_{\pi}$ with respect to the pairing

$$
V_{\pi} \times V_{\pi} \longrightarrow \mathbb{C}
$$




$$
\left(\phi_{1}, \phi_{2}\right) \longmapsto \int_{G(F) \backslash \backslash^{1} G\left(\mathbb{A}_{F}\right)} \phi_{1}(x) \overline{\phi_{2}(x)} d x
$$

with $d x$ the Tamagawa measure. Then each $m_{\pi} \pi\left(f^{1}\right)$ is an integral operator in $V_{\pi}$ with $L^{2}$-kernel

$$
K_{\pi\left(f^{1}\right)}(x, y)=\sum_{\phi \in \mathcal{B}_{\pi}}\left(\pi\left(f^{1}\right) \phi\right)(x) \overline{\phi(y)} .
$$

We note that, a priori, this sum only converges in $L_{0}^{2}\left(G(F) \backslash^{1} G\left(\mathbb{A}_{F}\right) \times G(F) \backslash \backslash^{1} G\left(\mathbb{A}_{F}\right)\right.$ ). However, Arthur has shown that there exists a (unique) square-integrable function that is smooth in $x$ and $y$ separately and represents this kernel [A1, Lemma 4.5 and Lemma 4.8]. We will denote this function by $K_{\pi\left(f^{1}\right)}(x, y)$.

We define

$$
\operatorname{TRT}_{G^{\sigma}, G^{\theta}}^{\mu}\left(\pi\left(f^{1}\right)\right):=\iint_{G^{\sigma}(F) \times G^{\theta}(F) \backslash^{2} G^{\sigma}\left(\mathbb{A}_{F}\right) \times^{2} G^{\theta}\left(\mathbb{A}_{F}\right)} \mu\left(x, y^{-1}\right) K_{\pi\left(f^{1}\right)}(x, y) d x d y .
$$

The integrand is smooth separately in $x$ and $y$ and is in the cuspidal subspace, hence rapidly decreasing by $[\mathrm{HC}, \S 4]$. Therefore it is absolutely convergent by [AGR, §2 Proposition 1]. Let $K_{\infty}$ be a maximal compact subgroup of $G\left(\mathbb{A}_{F, \infty}\right)$. We note that if $f^{1}$ is $K_{\infty}$-finite, then the sum over $\phi$ in (5.4) can be taken to be a finite sum over $K_{\infty}$-finite functions [A1, Above Lemma 4.5]. Therefore, in this case

$$
\operatorname{TRT}_{G^{\sigma}, G^{\theta}}^{\mu}\left(\pi\left(f^{1}\right)\right)=\sum_{\phi \in \mathcal{B}_{\pi}} \mathcal{P}_{\mu_{1}}^{\sigma}\left(\pi\left(f^{1}\right) \phi\right) \overline{\mathcal{P}_{\mu_{2}}^{\theta}(\phi)} .
$$

In general, if $\operatorname{TRT}_{G^{\sigma}, G^{\theta}}^{\mu}\left(\pi\left(f^{1}\right)\right) \neq 0$, then $\pi$ is both $\left(G^{\sigma}, \mu_{1}\right)$ and $\left(G^{\theta}, \mu_{2}\right)$-distinguished.

If $R\left(f^{1}\right)$ has image in $L_{0}^{2}\left(G(F) \backslash^{1} G\left(\mathbb{A}_{F}\right)\right)$, its kernel has $L^{2}$-expansion

$$
K_{f^{1}}(x, y)=\sum_{\pi} K_{\pi\left(f^{1}\right)}(x, y),
$$

where the sum is over equivalence classes of automorphic representations $\pi$ of ${ }^{1} G\left(\mathbb{A}_{F}\right)$ admitting a realization in $R_{0}$. Again, this kernel is represented by a (unique) square integrable function that is smooth in $x$ and $y$ separately [A1, Lemma 4.5 and Lemma 4.8]. We denote this function by $K_{f^{1}}(x, y)$ as well. Then, by [A1, §4], the equation in (5.6) holds pointwise.

Proposition 5.2. Let $f^{1} \in C_{c}^{\infty}\left({ }^{1} G\left(\mathbb{A}_{F}\right)\right)$ be defined as (4.5). Assume that the integral operator $R\left(f^{1}\right)$ has image in $L_{0}^{2}\left(G(F) \backslash{ }^{1} G\left(\mathbb{A}_{F}\right)\right)$. Then we have that

$$
\iint_{G^{\sigma}(F) \times G^{\theta}(F) \backslash^{2} G^{\sigma}\left(\mathbb{A}_{F}\right) \times{ }^{2} G^{\theta}\left(\mathbb{A}_{F}\right)} \mu\left(x, y^{-1}\right) K_{f^{1}}(x, y) d x d y=\sum_{\pi} \operatorname{TRT}_{G^{\sigma}, G^{\theta}}^{\mu}\left(\pi\left(f^{1}\right)\right),
$$

where the sum on the right is over equivalence classes of irreducible automorphic representations $\pi$ of ${ }^{1} G\left(\mathbb{A}_{F}\right)$ occurring in $R_{0}$. The integrand is absolutely integrable and the sum is absolutely convergent.

Proof. As noted above, $K_{f^{1}}(x, y)$ is a smooth function that is (by definition) in the closed subspace $L_{0}^{2}\left(G(F) \backslash{ }^{1} G\left(\mathbb{A}_{F}\right) \times G(F) \backslash{ }^{1} G\left(\mathbb{A}_{F}\right)\right)$. It is therefore rapidly decreasing 
[HC, $\S 4]$. Thus, by [AGR, $\S 2$, Proposition 1] the integral on the left in (5.7) is absolutely convergent. Formally, we have that

$$
\begin{aligned}
& \iint_{G^{\sigma}(F) \times G^{\theta}(F) \backslash^{2} G^{\sigma}\left(\mathbb{A}_{F}\right) \times^{2} G^{\theta}\left(\mathbb{A}_{F}\right)} \mu\left(x, y^{-1}\right) K_{f^{1}}(x, y) d x d y \\
& =\iint_{G^{\sigma}(F) \times G^{\theta}(F) \backslash^{2} G^{\sigma}\left(\mathbb{A}_{F}\right) \times^{2} G^{\theta}\left(\mathbb{A}_{F}\right)} \mu\left(x, y^{-1}\right) \sum_{\pi} K_{\pi\left(f^{1}\right)}(x, y) d x d y \\
& =\sum_{\pi} \iint_{G^{\sigma}(F) \times G^{\theta}(F) \backslash^{2} G^{\sigma}\left(\mathbb{A}_{F}\right) \times^{2} G^{\theta}\left(\mathbb{A}_{F}\right)} \mu\left(x, y^{-1}\right) K_{\pi\left(f^{1}\right)}(x, y) d x d y \\
& =\sum_{\pi} \operatorname{TRT}_{G^{\sigma}, G^{\theta}}^{\mu}\left(\pi\left(f^{1}\right)\right) .
\end{aligned}
$$

In order to make this rigorous, we must justify switching the sum and integral. As noted above, $K_{f^{1}}(x, y)=\sum_{\pi} K_{\pi\left(f_{1}\right)}(x, y)$ pointwise. By the Lebesgue dominated convergence theorem it suffices to prove that $\sum_{\pi}\left|K_{\pi\left(f^{1}\right)}(x, y)\right|$ is integrable over $G^{\sigma}(F) \times$ $G^{\theta}(F) \backslash{ }^{2} G^{\sigma}\left(\mathbb{A}_{F}\right) \times{ }^{2} G^{\theta}\left(\mathbb{A}_{F}\right)$.

Choose an integer $m>0$. If $m$ is sufficiently large, then there exists $K_{\infty}$-finite functions $g_{i} \in C_{c}^{m}\left({ }^{1} G\left(\mathbb{A}_{F}\right)\right)$ and an element $Z$ in the universal enveloping algebra $\mathfrak{U}\left(\operatorname{Lie}\left({ }^{1} G\left(\mathbb{A}_{F, \infty}\right)\right) \otimes \mathbb{C}\right)$ fixed by $K_{\infty}\left(\right.$ ?) with the following property: If $r_{0}>\operatorname{deg}(Z)$ and $h \in C_{c}^{r_{0}}\left({ }^{1} G\left(\mathbb{A}_{F}\right)\right)$ we can write

$$
h=\sum_{i=1}^{2} h_{i} * g_{i},
$$

where $h_{1}=h * Z$ and $h_{2}=h$ [A1, Corollary 4.2]. Notice that the $h_{i}$ are $K_{\infty}$-finite if $h$ is $K_{\infty}$-finite.

Write

$$
g^{*}(x): \overline{g\left(x^{-1}\right)}
$$

for compactly supported continuous functions $g \in C_{c}\left({ }^{1} G\left(\mathbb{A}_{F}\right)\right)$. Assume for the moment that $h \in C_{c}^{r}\left({ }^{1} G\left(\mathbb{A}_{F}\right)\right)$ is $K_{\infty}$-finite for some $r \geq r_{0}$. Applying the Cauchy-Schwarz inequality several times we have

$$
\begin{aligned}
\left|K_{\pi(h)}(x, y)\right| & =\left|\sum_{\phi \in \mathcal{B}_{\pi}}(\pi(h) \phi)(x) \overline{\phi(y)}\right|=\left|\sum_{i=1}^{2} \sum_{\phi \in \mathcal{B}_{\pi}}\left(\pi\left(h_{i}\right) \phi\right)(x) \overline{\left(\pi\left(g_{i}^{*}\right) \phi\right)(y)}\right| \\
& \leq \sum_{i=1}^{2}\left|\sum_{\phi \in \mathcal{B}_{\pi}}\left(\pi\left(h_{i}\right) \phi\right)(x)\right|\left|\sum_{\phi \in \mathcal{B}_{\pi}} \overline{\left(\pi\left(g_{i}^{*}\right) \phi\right)(y)}\right| \\
& \leq \sum_{i=1}^{2}\left(\sum_{\phi \in \mathcal{B}_{\pi}}\left|\left(\pi\left(h_{i}\right) \phi\right)(x)\right|\right)\left(\sum_{\phi \in \mathcal{B}_{\pi}}\left|\left(\pi\left(g_{i}^{*}\right) \phi\right)(y)\right|\right) \\
& =\sum_{i=1}^{2} K_{\pi\left(h_{i} * h_{i}^{*}\right)}(x, x) K_{\pi\left(g_{i}^{*} * g_{i}\right)}(y, y) .
\end{aligned}
$$


Here the $\phi \in \mathcal{B}_{\pi}$ are assumed to be $K_{\infty}$-finite (and hence smooth) and the sums can be taken to be finite because we have assumed that $h$ is $K_{\infty}$-finite. We note that $K_{\pi\left(h_{i} * h_{i}^{*}\right)}(x, x)$ and $K_{\pi\left(g_{i}^{*} * g_{i}\right)}(y, y)$ are nonnegative functions. Thus, applying the Cauchy-Schwarz inequality again we have

$$
\begin{aligned}
\sum_{\pi}\left|K_{\pi(h)}(x, y)\right| & \leq \sum_{\pi} \sum_{i=1}^{2} K_{\pi\left(h_{i} * h_{i}^{*}\right)}(x, x) K_{\pi\left(g_{i}^{*} * g_{i}\right)}(y, y) \\
& \leq \sum_{i=1}^{2}\left(\sum_{\pi} K_{\pi\left(h_{i} * h_{i}^{*}\right)}(x, x)\right)\left(\sum_{\pi} K_{\pi\left(g_{i}^{*} * g_{i}\right)}(y, y)\right) .
\end{aligned}
$$

We claim that the estimate (5.8) is valid without assuming that $h$ is $K_{\infty}$-finite. For each $r$, give $C_{c}^{r}\left({ }^{1} G\left(\mathbb{A}_{F}\right)\right)$ the topology explained in $[\mathrm{A} 1, \S 2]$. Then there exists a continuous seminorm $\|\cdot\|_{0}$ on $C_{c}\left({ }^{1} G\left(\mathbb{A}_{F}\right)\right)$ and a positive integer $N$ depending only on $G$ such that for each $K_{\infty}$-finite $h \in C_{c}^{r}\left({ }^{1} G\left(\mathbb{A}_{F}\right)\right)$ with $r \geq r_{0}$ we have

$$
\left(\sum_{\pi} K_{\pi\left(h_{i} * h_{i}^{*}\right)}(x, x)\right)\left(\sum_{\pi} K_{\pi\left(g_{i}^{*} * g_{i}\right)}(y, y)\right) \leq\left\|h_{i} * h_{i}^{*}\right\|_{0}^{1 / 2}\left\|g_{i}^{*} * g_{i}\right\|_{0}^{1 / 2}\|x\|^{N}\|y\|^{N}
$$

and hence

$$
\sum_{\pi}\left|K_{\pi(h)}(x, y)\right| \leq \sum_{i=1}^{2}\left\|h_{i} * h_{i}^{*}\right\|_{0}^{1 / 2}\left\|g_{i}^{*} * g_{i}\right\|_{0}^{1 / 2}\|x\|^{N}\|y\|^{N}
$$

(see [A1, Proof of Lemma 4.4]). Here $\|\cdot\|$ is the height function on ${ }^{1} G\left(\mathbb{A}_{F}\right)$ given in $[A 1, \S 2]$. It follows that for fixed $(x, y)$ the real-valued functions

$$
h \mapsto \sum_{\pi}\left|K_{\pi(h)}(x, y)\right| \text { and } h \mapsto \sum_{i=1}^{2}\left(\sum_{\pi} K_{\pi\left(h_{i} * h_{i}^{*}\right)}(x, x)\right)\left(\sum_{\pi} K_{\pi\left(g_{i}^{*} * g_{i}\right)}(y, y)\right)
$$

are continuous on the $K_{\infty}$-finite subset of $C_{c}^{r}\left({ }^{1} G\left(\mathbb{A}_{F}\right)\right)$. If they admitted continuous extensions to $C_{c}^{r}\left({ }^{1} G\left(\mathbb{A}_{F}\right)\right)$, then our claim would be proven. Due to the fact that $K_{\infty}$-finite functions in $C_{c}^{r}\left({ }^{1} G\left(\mathbb{A}_{F}\right)\right)$ are not in general dense in $C_{c}^{r}\left({ }^{1} G\left(\mathbb{A}_{F}\right)\right)$, we are not sure whether this statement is true [A1, p. 931]. Instead, following Arthur, we view a general $h \in C_{c}^{r}\left({ }^{1} G\left(\mathbb{A}_{F}\right)\right)$ as an element of $C_{c}^{r-l_{0}}\left({ }^{1} G\left(\mathbb{A}_{F}\right)\right)$ and approximate it in $C_{c}^{r-l_{0}}\left({ }^{1} G\left(\mathbb{A}_{F}\right)\right)$ with a sequence of $K_{\infty}$-finite functions. Here $l_{0}$ is a certain positive integer. This will allow us to deduce the claim.

Let $x$ and $y$ be fixed and let $h \in C_{c}^{r}\left({ }^{1} G\left(\mathbb{A}_{F}\right)\right)$; we do not assume $h$ is $K_{\infty}$-finite. We assume that $r>l_{0}$, where $l_{0}$ is the integer discussed after the proof of [A1, Lemma 4.4]; it depends only on $G$. For each such $h$ there is a countable set of $K_{\infty}$-finite functions $h_{\omega} \in C_{c}^{r-l_{0}}\left({ }^{1} G\left(\mathbb{A}_{F}\right)\right)$ such that $\sum_{\omega} h_{\omega}$ converges absolutely to $h$ in $C_{c}^{r-l_{0}}\left({ }^{1} G\left(\mathbb{A}_{F}\right)\right)$ and if $\|\cdot\|_{\text {? }}$ is any continuous seminorm on $C_{c}^{r-l_{0}}\left({ }^{1} G\left(\mathbb{A}_{F}\right)\right)$ then

$$
f \mapsto \sum_{\omega}\left\|f_{\omega}\right\|_{?}
$$


is again a continuous seminorm on $C_{c}^{r}\left({ }^{1} G\left(\mathbb{A}_{F}\right)\right.$ ) (see after the proof of $[\mathrm{A} 1$, Lemma 4.4]). Applying this with

$$
\|h\|_{r_{0}}=\sum_{i=1}^{2}\left\|h_{i} * h_{i}^{*}\right\|_{0}^{1 / 2}\left\|g_{i}^{*} * g_{i}\right\|_{0}^{1 / 2}\|x\|^{N}\|y\|^{N}
$$

implies that the functions in (5.9) extend to define continuous functions on $C_{c}^{r-l_{0}}\left({ }^{1} G\left(\mathbb{A}_{F}\right)\right)$. This implies our claim that (5.8) is valid for all $h \in C_{c}^{r-l_{0}}\left({ }^{1} G\left(\mathbb{A}_{F}\right)\right)$.

Choose a positive integer $L>0$. Enlarging $m$ if necessary in order to make $g_{1} \in$ $C_{c}^{m}\left({ }^{1} G\left(\mathbb{A}_{F}\right)\right)$ smoother, we apply the proof of $[\mathrm{A} 3$, Theorem $14.1(\mathrm{a})]$ (see also [A2, §2]) to conclude that

$$
\begin{gathered}
\sum_{\pi}\left|\Lambda_{2}^{T} K_{\pi\left(h_{i} * h_{i}^{*}\right)}(x, x)\right| \leq C\left(h_{i}\right)\|x\|^{-L} \text { and } \\
\sum_{\pi}\left|\Lambda_{2}^{T} K_{\pi\left(g_{i}^{*} g_{i}\right)}(y, y)\right| \leq C\left(g_{i}\right)\|y\|^{-L},
\end{gathered}
$$

where $\Lambda_{2}^{T}$ is Arthur's truncation operator (see [A2, §1]), $C\left(h_{i}\right), C\left(g_{i}\right)$ are constants, and $T$ is assumed to be sufficiently regular. On the other hand,

$$
\Lambda_{2}^{T} K_{\pi\left(h_{i} * h_{i}^{*}\right)}(x, x)=K_{\pi\left(h_{i} * h_{i}^{*}\right)}(x, x), \quad \Lambda_{2}^{T} K_{\pi\left(g_{i}^{*} * g_{i}\right)}(y, y)=K_{\pi\left(g_{i}^{*} * g_{i}\right)}(y, y)
$$

for unitary $\pi$ realized in $L_{0}^{2}\left(G(F) \backslash{ }^{1} G\left(\mathbb{A}_{F}\right)\right)[$ A2, §1]. Thus

$$
\sum_{\pi} K_{\pi\left(h_{i} * h_{i}^{*}\right)}(x, x) \text { and } \sum_{\pi} K_{\pi\left(g_{i} * g_{i}^{*}\right)}(y, y)
$$

are both rapidly decreasing. Applying the proof of [AGR, $\S 2$ Proposition 1] we see that

$$
\iint_{G^{\sigma}(F) \times G^{\theta}(F) \backslash^{2} G^{\sigma}\left(\mathbb{A}_{F}\right) \times^{2} G^{\theta}\left(\mathbb{A}_{F}\right)}\left(\sum_{\pi} K_{\pi\left(h_{i} * h_{i}^{*}\right)}(x, x)\right)\left(\sum_{\pi} K_{\pi\left(g_{i}^{*} * g_{i}\right)}(y, y)\right) d x d y<\infty .
$$

In view of (5.8) an application of the Lesbesgue dominated convergence theorem completes the proof of the proposition.

Lemma 5.3. Suppose that $f=\otimes_{v}^{\prime} f_{v} \in C_{c}^{\infty}\left(G\left(\mathbb{A}_{F}\right)\right)$. If $f_{v}$ is F-supercuspidal for some finite place $v$, then $R\left(f^{1}\right)$ has image in $L_{0}^{2}\left(G(F) \backslash{ }^{1} G\left(\mathbb{A}_{F}\right)\right)$.

Proof. For any $\psi \in L^{2}\left(G(F) \backslash{ }^{1} G\left(\mathbb{A}_{F}\right)\right)$, and for the unipotent radical $N$ of any proper parabolic subgroup of $G$, we have that

$$
\begin{aligned}
& \int_{N(F) \backslash N\left(\mathbb{A}_{F}\right)} R\left(f^{1}\right) \psi\left(n^{-1} x\right) d n \\
& =\int_{N(F) \backslash N\left(\mathbb{A}_{F}\right)}\left(\int_{N(F) \backslash{ }^{1} G\left(\mathbb{A}_{F}\right)} \sum_{y \in N(F)} f^{1}\left(x^{-1} n y g\right) \psi(g) d g\right) d n \\
& =\int_{N(F) \backslash{ }^{1} G\left(\mathbb{A}_{F}\right)}\left(\int_{N\left(\mathbb{A}_{F}\right)} f^{1}\left(x^{-1} n g\right) d n\right) \psi(g) d g=0,
\end{aligned}
$$

since we have that

$$
\int_{N\left(\mathbb{A}_{F}\right)} f^{1}\left(x^{-1} n g\right) d n=\prod_{v \mid \infty} \int_{N\left(F_{v}\right)} f_{v}^{1}\left(x_{v}^{-1} n_{v} g_{v}\right) d n_{v} \prod_{v \nmid \infty} \int_{N\left(F_{v}\right)} f_{v}\left(x_{v}^{-1} n_{v} g_{v}\right) d n_{v}=0
$$


for all $x \in{ }^{1} G\left(\mathbb{A}_{F}\right)$.

We now restate and prove the main theorem of this paper.

Theorem 5.4. Suppose that $G^{\sigma}$ is connected. Let $f=\otimes_{v} f_{v} \in C_{c}^{\infty}\left(G\left(\mathbb{A}_{F}\right)\right)$ be such that there exist places $v_{1}, v_{2}, v_{3}$ of $F$ (not necessarily distinct) such that

(1) $f_{v_{1}}$ is supported on relatively $\tau$-elliptic elements of $G\left(F_{v_{1}}\right)$,

(2) $f_{v_{2}}$ is supported on strongly relatively $\tau$-regular elements of $G\left(F_{v_{2}}\right)$,

(3) $f_{v_{3}}$ is F-supercuspidal.

Then we have that

$$
\sum_{\{\gamma\}} c^{\tau}(\gamma) \operatorname{TRO}_{\gamma}^{\mu}(f)=\sum_{\pi} \operatorname{TRT}_{G^{\sigma}, G^{\theta}}^{\mu}\left(\pi\left(f^{1}\right)\right),
$$

where the sum on the left is over relevant strongly relatively $\tau$-regular elliptic classes in $G(F)$ and the sum on the right is over equivalence classes of automorphic representations $\pi$ of ${ }^{1} G\left(\mathbb{A}_{F}\right)$ admitting a realization in $L_{0}^{2}\left(G(F) \backslash{ }^{1} G\left(\mathbb{A}_{F}\right)\right)$.

Proof. Note that $K_{f^{1}}^{e}(x, y)=K_{f^{1}}(x, y)$ by our assumptions on support of $f$. Hence the theorem follows immediately from Theorem 4.1 and Proposition 5.2.

\section{ACKNOWLEDGMENTS}

I would like to thank the referees for their useful comments and corrections. I also thank Jayce Getz for his encouragement and helpful comments.

\section{REFERENCES}

[A1] J. Arthur, A trace formula for reductive groups I: Terms associated to classes in $G(\mathbb{Q})$, Duke Math. J. 45 No. 4 (1978), 911-952. 13, 14, 15, 16

[A2] J. Arthur, A trace formula for reductive groups II: Applications of a truncation operator, Compositio Math. 40 (1980), 87-121. 16

[A3] J. Arthur, An introduction to the trace formula, in Harmonic Analysis, The Trace Formula, and Shimura Varieties, Clay Mathematics Proceedings 4 2005. 16

[AGR] A. Ash, D. Ginzburg, and S. Rallis, Vanishing periods of cusp forms over modular symbols, Math. Ann. 296 No. 4 (1993), 709-723. 2, 12, 13, 14, 16

[BR] P. Bardsley and R. W. Richardson, Étale Slices for algebraic transformation groups in characteristic p, Proc. London Math. Soc. 51 No 3 (1985), 295-317 5

[BDKV] J. Bernstein, P. Deligne, D. Kazhdan, and M.-F. Vigneras, Representations des groupes sur un corps local, Hermann, Paris, 1984. 1, 2

[BLR] S. Bosch, W. Lütkebohmert, and M. Raynaud, Néron Models, Springer-Verlag, NY, 1990. 8

[F] Y. Flicker, Relative trace formula and simple algebras, Proc. AMS 99 No. 3 (1987), 421-426. 4

[G] S. Gelbart, Lectures on the Aurthur-Selberg trace formula, Univ. Lecture series of AMS 9, Providence, RI, 1996. 12

[GW] J. Getz and E. Wambach, Twisted relative trace formulae with a view towards unitary groups, submitted. 1, 2, 4

[HM] J. Hakim and F. Murnaghan, Globalization of distinguished supercuspidal representations of GL(n), Canad. Math. Bull. 45 (2) (2002), 220-230. 1

[HC] Harish-Chandra, Automorphic forms on semisimple Lie groups, Lecture Notes in Mathematics 62, Springer-Verlag, 1968 13, 14

[HW] A. G. Helminck and S. P. Wang, Rationality properties of involutions of reductive groups, Advances in Math. 99 (1993), 26-97. 4 
[JL] H. Jacquet and K. F. Lai, A relative trace formula, Compositio Math. 54 No. 2 (1985), 243-310. 4

[L] J-P. Labesse, Cohomologie, stabilisation at changement de base, Astérisque 257 1999. 11, 12

[GIT] D. Mumford, J. Fogarty, and F. Kirwan, Geometric invariant theory, Springer, New York, 2002. 7,11

[RR] C. Rader and S. Ralls, Spherical characters on $\mathfrak{p}$-adic symmetric spaces, American J. Math. 118 (1996), 91-178 6

[Ri] R. W. Richardson, Orbits, invariants and representations associated to involutions of reductive groups, Invent. Math. 66 (1982), 287-312. 4, 5

[Ro] J. D. Rogawski, Representations of $G L(n)$ and division algebras over a p-adic field, Duke Math. J. 50 No. 1 (1983), 161-196. 1

[S1] R. Steinberg, Regular elements of semisimple algebraic groups, Publ. Math. d' l'IHÉS 25 (1965), 49-80. 8

[S2] R. Steinberg, Endomorphisms of linear algebraic groups, Memoirs of the AMS 80, AMS, Providence, RI, 1968. 1

[T] J. Tits, Reductive groups over local fields, Automorphic forms, representations and $L$-functions, Proc. Symp. in Pure Math. 33, AMS, Providence RI, 1979. 7

Department of Mathematics and Statistics, University at Albany, Albany, NY 12222

E-mail address: hhahn@albany.edu 